\title{
Classification of Arrangements by the Number of Their Cells
}

\author{
Nicola Martinov \\ ul. Tintjava No. 20, Bloc 3, Vhod D, \\ Sofia 1113, Bulgaria
}

\begin{abstract}
The possible pairs ( $n, f)$ of integers for which there are arrangements with $n$ lines and $f$ cells are determined. The pair $(n, f)$ corresponds to an arrangement if and only if there is an integer $k$ with $0 \leq k \leq n-2$ such that
\end{abstract}

$$
(n-k)(k+1)+\left(\begin{array}{l}
k \\
2
\end{array}\right)-\min \left\{n-k,\left(\begin{array}{l}
k \\
2
\end{array}\right)\right\} \leq f \leq(n-k)(k+1)+\left(\begin{array}{l}
k \\
2
\end{array}\right) .
$$

\section{Introduction and Terminology}

We follow the terminology of Grünbaum [4], with minor modifications. By arrangement $\mathscr{A}$ of lines we mean a finite family of $n(\mathscr{A}) \geq 2$ distinct lines in the real projective plane $\mathscr{P}$. We associate with the arrangement $\mathscr{A}$ the two-dimensional cell complex obtained by the decomposition of $\mathscr{P}$ by the lines of $\mathscr{A}$; the vertices, edges, and cells (polygonal regions) of that complex are said to be vertices, edges, and cells of the arrangement. The number of cells is denoted by $f(\mathscr{A})$. If $V$ is a vertex, then $t(V)$ is the number of lines incident with $V$. The maximal value of $t(V)$ in the arrangement $\mathscr{A}$ is denoted by $t(\mathscr{A})$. If $G$ is a line of the arrangement, then $r(G)$ is the number of vertices incident with $G$. The maximal number of vertices incident with a line of $\mathscr{A}$ is denoted by $r(\mathscr{A})$. If $t(\mathscr{A})=2$, then $\mathscr{A}$ is said to be a simple arrangement, and if $t(\mathscr{A})=n(\mathscr{A})=n$, then $\mathscr{A}$ is said to be an $n$-pencil.

It is well known that for each arrangement $\mathscr{A}$ we have

$$
n \leq f(\mathscr{A}) \leq\left(\begin{array}{l}
n \\
2
\end{array}\right)+1,
$$

where $n=n(\mathscr{A})$. Moreover, equality on the left characterizes pencils, and equality on the right characterizes simple arrangements. 
Those numbers in the inverval

$$
\mathscr{I}=\left[n,\left(\begin{array}{l}
n \\
2
\end{array}\right)+1\right]
$$

which cannot be values of $f(\mathscr{A})$ are said to be inaccessible. It is easily seen that the intervals $\mathscr{I}_{1}=[n+1,2 n-3]$ for $n \geq 4$, and $\mathscr{I}_{2}=[2 n-1,3 n-7]$, for $n \geq 6$, consist only of inaccessible numbers. Conjecture 2.4 of [4], according to which the interval $\mathscr{I}_{3}=[3 n-4,4 n-13]$ for $n \geq 9$ also consists only of inaccessible numbers, has been proved earlier, by Cordovil [1], Martinov [5], and (for $n \geq 40$ ) Purdy [6]. The aim of this note can also be described as finding all the inaccessible numbers, for all $n$.

As the reader will note, the proofs are purely combinatorial; hence they apply equally well to arrangements of pseudolines, that is, to oriented matroids of rank 3 .

The results on arrangements directly transfer to zonohedra. A zonohedron is a convex polyhedron, each of whose faces is centrally symmetric (and therefore the polyhedron is centrally symmetric as well). Zonohedra were introduced by the Russian crystallographer Fedorov [3] (although his definition includes a broader class of polyhedra, see [8]). A zone of a zonohedron is every cycle of faces in which the successive faces are adjacent and meet in parallel edges. It is well known [2] that arrangements of lines are combinatorially equivalent to zonohedra, the lines, cells, and vertices of arrangements corresponding to zones, pairs of opposite vertices, and pairs of opposite faces of zonohedra.

Our results are somewhat analogous to those of Salamon and Erdös [7] concerning the problem of determining all possible pairs $(n, v)$, where $v$ is the number of vertices of an arrangement with $n$ lines.

\section{Preliminary Results}

Let $p, q$, and $m$ be nonnegative integers and let

$$
m \leq \min \left\{p,\left(\begin{array}{l}
q \\
3
\end{array}\right)\right\}
$$

Let us denote by $\mathbb{B}_{p, q}^{m}$ the family of the arrangements, each of which is a union of a $p$-pencil and a simple arrangement of $q$ lines different from the ones in the pencil and having $m$ of their $\left(\begin{array}{l}q \\ 2\end{array}\right)$ intersection points incident with lines of the pencil.

It is easy to verify the following lemmas:

Lemma 1. If the arrangement $\mathscr{A}$ is not a pencil and if $n(\mathscr{A})=n \geq 9$ and $f(\mathscr{A}) \leq 3 n+5$, then

$$
\mathscr{A} \in \mathbb{B}_{n-1,1}^{0} \cup \mathbb{B}_{n-2,2}^{0} \cup \mathbb{B}_{n-2,2}^{1}
$$


A straightforward calculation shows

Lemma 2. If $\mathscr{A} \in \mathbb{B}_{p, q}^{m}$, then

$$
f(\mathscr{A})=p(q+1)+\left(\begin{array}{l}
q \\
2
\end{array}\right)-m .
$$

As mentioned above, the results of [1] and [5], establishing the validity of Grünbaum's conjecture, imply

Lemma 3. If $\mathscr{A}$ is an arrangement with $f(\mathscr{A})<4 n(\mathscr{A})-12$, then $f(\mathscr{A}) \leq$ $3 n(\mathscr{A})-5$.

We also need:

Lemma 4. If the arrangement $\mathscr{A}$ is not a pencil and if $n(\mathscr{A}) \geq 10$, then $r(\mathscr{A}) \geq 5$.

Proof. 1. Let $f(\mathscr{A})<4 n(\mathscr{A})-12$. By Lemma 3 we have $f(\mathscr{A}) \leq 3 n(\mathscr{A})-5$. The last inequality and Lemma 1 imply $t(\mathscr{A}) \geq n(\mathscr{A})-2 \geq 8$. Therefore $r(\mathscr{A}) \geq t(\mathscr{A}) \geq 8$.

2. Let $f(\mathscr{A}) \geq 4 n(\mathscr{A})-12$. Since, for the number of edges, we have $f_{1}(\mathscr{A}) \geq$ $\frac{3}{2} f(\mathscr{A})$, and since $r(\mathscr{A}) \geq f_{1}(\mathscr{A}) / n(\mathscr{A})$, we have

$$
r(\mathscr{A}) \geq] \frac{f_{1}(\mathscr{A})}{n(\mathscr{A})}[\geq] 6-\frac{18}{10}[=5 .
$$

\section{Main Results}

Theorem 1. Let $k$ be an integer, $k \geq 3$. The following statements are valid for each arrangement $\mathscr{A}$, with

$$
n(\mathscr{A}) \geq\left(\begin{array}{c}
k+1 \\
2
\end{array}\right)+3
$$

which is not a pencil:

$\mathrm{A}_{k}$. If

$$
f(\mathscr{A}) \leq k n(\mathscr{A})-\left(\begin{array}{c}
k+1 \\
2
\end{array}\right)+1,
$$

then $t(\mathscr{A}) \geq n(\mathscr{A})-k+1$.

$\mathrm{B}_{k} \cdot r(\mathscr{A}) \geq k+\frac{1}{2}(k-3)+15 /\left(k^{2}+k+6\right)$.

$\mathrm{C}_{k}$. If $f(\mathscr{A})<(k+1)(n(\mathscr{A})-k)$, then

$$
f(\mathscr{A}) \leq k n(\mathscr{A})-\left(\begin{array}{c}
k+1 \\
2
\end{array}\right)+1 .
$$


Proof. We use induction on $k$. The theorem is valid for $k=3$. $\mathrm{A}_{3}$ follows directly from Lemma 1 ; if $\mathscr{A}$ is not a pencil and has a least seven lines, then clearly $r(\mathscr{A}) \geq 4$, hence $B_{3}$ holds; statement $C_{3}$ coincides with Lemma 3 . We now assume that $k \geq 3$ and that $A_{k-1}, B_{k-1}$, and $C_{k-1}$ are valid for each arrangement $\mathscr{A}^{*}$ which is not a pencil and satisfies

$$
n\left(\mathscr{A}^{*}\right) \geq\left(\begin{array}{c}
k-1 \\
2
\end{array}\right)+3
$$

When $\mathscr{A}^{*}$ is obtained from $\mathscr{A}$ by removing a line and $f(\mathscr{A})>2 n(\mathscr{A})-2$, these conditions are satisfied; only this case occurs in what follows.

Proof of $\mathrm{A}_{k} . \quad$ If $f(\mathscr{A})<k(n(\mathscr{A})-k+1)$, then by $\mathrm{C}_{k-1}$ we have

$$
f(\mathscr{A}) \leq(k-1) n(\mathscr{A})-\left(\begin{array}{l}
k \\
2
\end{array}\right)+1
$$

and by $\mathrm{A}_{k-1}$ it follows that $t(\mathscr{A}) \geq n(\mathscr{A})-k+2$. Hence we have to prove $\mathrm{A}_{k}$ for $f(\mathscr{A})=k(n(\mathscr{A})-k+1)+m$, where

$$
0 \leq m \leq\left(\begin{array}{c}
k-1 \\
2
\end{array}\right)
$$

We use induction on $m$. Let $G$ be a line of $\mathscr{A}$ with $r(G)=r(\mathscr{A})$. From $\mathrm{B}_{k-1}$ (when $k>4$ ) and Lemma 4 (when $k=4$ ), it follows that $r(\mathscr{A}) \geq k+1$. Hence for the arrangement $\mathscr{A}^{*}=\mathscr{A} \backslash G$ we have

$f\left(\mathscr{A}^{*}\right)=f(\mathscr{A})-r(\mathscr{A}) \leq k(n(\mathscr{A})-k+1)+m-k-1=k(n(\mathscr{A})-k)+m-1$

1. Let $m=0$. Then (1) implies $f\left(\mathscr{A}^{*}\right) \leq k\left(n\left(\mathscr{A}^{*}\right)-k+1\right)$, and according to $\mathrm{C}_{k-1}$ we have

$$
f\left(\mathscr{A}^{*}\right) \leq(k-1) n\left(\mathscr{A}^{*}\right)-\left(\begin{array}{l}
k \\
2
\end{array}\right)+1
$$

hence $\mathrm{A}_{k-1}$ implies that $t\left(\mathscr{A}^{*}\right) \geq n\left(\mathscr{A}^{*}\right)-k+2$, which means that $t(\mathscr{A}) \geq$ $n(\mathscr{A})-k+1$.

2. Now let $m>0$, and assume $A_{k}$ is valid for smaller values of $m$. Then the inductive assumption and (1) imply $t\left(\mathscr{A}^{*}\right) \geq n\left(\mathscr{A}^{*}\right)-k+1$ and $t(\mathscr{A}) \geq n(\mathscr{A})-k$. Therefore $r(\mathscr{A}) \geq t(\mathscr{A}) \geq n(\mathscr{A})-k$, and we obtain the following estimate of $f\left(\mathscr{A}^{*}\right)$, which is more precise than (1):

$$
\begin{aligned}
f\left(\mathscr{A}^{*}\right) & =f(\mathscr{A})-r(\mathscr{A}) \leq k(n(\mathscr{A})-k+1)+m-n(\mathscr{A})+k \\
& =k n\left(\mathscr{A}^{*}\right)-k^{2}+2 k+m-n\left(\mathscr{A}^{*}\right) .
\end{aligned}
$$


However,

$$
n(\mathscr{A})-m \geq\left(\begin{array}{c}
k+1 \\
2
\end{array}\right)+3-\left(\begin{array}{c}
k-1 \\
2
\end{array}\right)=2 k+2 \text {. }
$$

Hence $f\left(\mathscr{A}^{*}\right)<k(n(\mathscr{A})-k+1)$, and using $\mathrm{C}_{k-1}$ we obtain

$$
f\left(\mathscr{A}^{*}\right) \leq(k-1) n\left(\mathscr{A}^{*}\right)-\left(\begin{array}{l}
k \\
2
\end{array}\right)+1
$$

Applying $\mathrm{A}_{k-1}$ we obtain $t\left(\mathscr{A}^{*}\right)-k+2$, which means that $t(\mathscr{A}) \geq n(\mathscr{A})-$ $k+1$.

Proof of $\mathrm{B}_{k}$. 1. Let

$$
f(\mathscr{A}) \leq k n(\mathscr{A})-\left(\begin{array}{c}
k+1 \\
2
\end{array}\right)+1
$$

Then, from statement $A_{k}$ already proved, we have

$$
t(\mathscr{A}) \geq n(\mathscr{A})-k+1 \geq\left(\begin{array}{c}
k+1 \\
2
\end{array}\right)+3-k+1=\left(\begin{array}{l}
k \\
2
\end{array}\right)+4 \text {. }
$$

Therefore $B_{k}$ is valid in this case.

2. Let

$$
f(\mathscr{A})>k n(\mathscr{A})-\left(\begin{array}{c}
k+1 \\
2
\end{array}\right)+1
$$

Since for the number of edges we have $f_{1}(\mathscr{A}) \geq \frac{3}{2} f(\mathscr{A})$ and $r(\mathscr{A}) \geq f_{1}(\mathscr{A}) / n(\mathscr{A})$, in this case we obtain

$$
r(\mathscr{A}) \geq \frac{3 f(\mathscr{A})}{2 n(\mathscr{A})} \geq \frac{3}{2} k-\frac{3\left(k^{2}+k-4\right)}{2\left(k^{2}+k+6\right)}=k+\frac{1}{2}(k-3)+\frac{15}{k^{2}+k+6}
$$

Proof of $\mathrm{C}_{k}$. Assuming $\mathrm{C}_{k}$ is false, let $\mathscr{A}$ be an arrangement with the minimal number $n=n(\mathscr{A})$ of lines such that

$$
k n-\left(\begin{array}{c}
k+1 \\
2
\end{array}\right)+1<f(\mathscr{A})<(k+1)(n-k) \text { and } n \geq\left(\begin{array}{c}
k+1 \\
2
\end{array}\right)+3
$$

Let $G$ be a line of $\mathscr{A}$ with $r(G)=r(\mathscr{A})$. Then by $\mathrm{B}_{k}$ we have $r(\mathscr{A}) \geq k+2$, and for the arrangement $\mathscr{A}^{*}=\mathscr{A} \backslash G$ we have

$$
f\left(\mathscr{A}^{*}\right)=f(\mathscr{A})-r(G)<(k+1)(n(\mathscr{A})-k)-(k+2)<(k+1)\left(n\left(\mathscr{A}^{*}\right)-k\right) .
$$


Now, if

$$
n(\mathscr{A})>\left(\begin{array}{c}
k+1 \\
2
\end{array}\right)+3
$$

then

$$
n\left(\mathscr{A}^{*}\right) \geq\left(\begin{array}{c}
k+1 \\
2
\end{array}\right)+3
$$

Since (2) is not valid for arrangements with fewer than $\left(\begin{array}{c}k+1 \\ 2\end{array}\right)+3$ lines, then

$$
f\left(\mathscr{A}^{*}\right) \leq k n\left(\mathscr{A}^{*}\right)-\left(\begin{array}{c}
k+1 \\
2
\end{array}\right)+1
$$

On the other hand, if

$$
n(\mathscr{A})=\left(\begin{array}{c}
k+1 \\
2
\end{array}\right)+3
$$

then (3) will again be valid because there will be only one integer in the interval

$$
\mathscr{I} k=\left(k n(\mathscr{A})-\left(\begin{array}{c}
k+1 \\
2
\end{array}\right)+1,(k+1)(n(\mathscr{A})-k)\right.
$$

since this integer is $f(\mathscr{A})$, we have $f\left(\mathscr{A}^{*}\right)<f(\mathscr{A})$.

From (3), by statement $\mathrm{A}_{k}$ proved previously, we have $t\left(\mathscr{A}^{*}\right) \geq n\left(\mathscr{A}^{*}\right)-k+1$, hence $t(\mathscr{A}) \geq n(\mathscr{A})-k$ and $r(\mathscr{A}) \geq n(\mathscr{A})-k$. From these we obtain the more precise estimate $f\left(\mathscr{A}^{*}\right)<(k+1)(n(\mathscr{A})-k)-n(\mathscr{A})+k=k(n(\mathscr{A})-k+1)$. Applying $\mathrm{C}_{n-1}$ we obtain

$$
f\left(\mathscr{A}^{*}\right) \leq(k-1) n\left(\mathscr{A}^{*}\right)-\left(\begin{array}{l}
k \\
2
\end{array}\right)+1
$$

and

$$
f(\mathscr{A}) \leq f\left(\mathscr{A}^{*}\right)+n(\mathscr{A})-1 \leq k n(\mathscr{A})-\left(\begin{array}{c}
k+1 \\
2
\end{array}\right)+1
$$

which contradicts (2).

We note that the theorem is also valid for $k=1,2,3$, since the intervals $\mathscr{I}_{1}$, $\mathscr{I}_{2}, \mathscr{I}_{3}$ consist only of inaccessible numbers. 
Corollary. If integers $n$ and $f$ satisfy

$$
k n-\left(\begin{array}{c}
k+1 \\
2
\end{array}\right)+1<f<(k+1)(n-k)
$$

for every natural $k$, then the pair $(n, k)$ does not correspond to an arrangement, that is, an arrangement with $n$ lines and $f$ cells does not exist.

Theorem 2. The pair ( $n, f)$ corresponds to an arrangement if and only if there exists an integer $k \leq n-2$ such that

$$
(n-k)(k+1)+\left(\begin{array}{l}
k \\
2
\end{array}\right)-\min \left\{n-k,\left(\begin{array}{l}
k \\
2
\end{array}\right)\right\} \leq f \leq(n-k)(k+1)+\left(\begin{array}{l}
k \\
2
\end{array}\right)
$$

Proof. Let $k$ be an integer from the interval $[1, n-2]$ and let

$$
m_{0}=\min \left\{n-k,\left(\begin{array}{l}
k \\
2
\end{array}\right)\right\}
$$

According to Lemma 2, the number of cells of arrangements in the class $\mathbb{B}_{n-k, k}^{m}$, where $m=0,1, \ldots, m_{0}$, is

$$
(n-k)(k+1)+\left(\begin{array}{l}
k \\
2
\end{array}\right)-m
$$

and therefore they yield all the integers of the interval $\mathscr{J}_{k}=\left[a_{k}, b_{k}\right]$, where

$$
a_{k}=(n-k)(k+1)+\left(\begin{array}{l}
k \\
2
\end{array}\right)-m_{0} \quad \text { and } \quad b_{k}=(n-k)(k+1)+\left(\begin{array}{l}
k \\
2
\end{array}\right)
$$

As $k$ grows from 1 to $n-2$, the interval $\mathscr{J}_{k}$ moves from the left end to the right end of the interval

$$
\mathscr{I}=\left[n,\left(\begin{array}{l}
n \\
2
\end{array}\right)+1\right]
$$

but, for $k>1$, we have

$$
\left[b_{k-1}, a_{k}\right]=\mathscr{I}_{k} \quad \text { if } \quad m_{0}=\left(\begin{array}{l}
n \\
2
\end{array}\right)
$$

and $a_{k}-b_{k-1}=-1$ if $m_{0}=n-k$. Therefore the intervals $\mathscr{I}_{k}$ cover all parts of the interval $\mathscr{I}$ except the subintervals $\mathscr{I}_{k}$, which proves Theorem 2 . 


\section{Acknowledgment}

I would like to thank the referee for his corrections of my syntactical and typographical errors, for indicating omissions in the text, and for providing some of the references.

\section{References}

1. R. Cordovil, Sur l'évaluation $t(M ; 2,0)$ du polynôme de Tutte d'un matrö̈de et une conjecture de B. Grünbaum rélative aux arrangements de droites du plan. European J. Combin. 1 (1980), 317-322.

2. H. S. M. Coxeter, The classification of zonohedra by means of projective diagrams. J. Math. Pures Appl. 41 (1962), 137-156 (reprinted with improvements in Twelve Geometric Essays, Southern Illinois University Press, Carbondale, IL, 1968).

3. E. S. Fedorov, Nachala Ucheniya o Figurah. Notices Imperial Petersburg Mineral. Soc. 2nd ser. 24 (1995), 1-279 (reprinted in the series Classics of Sciences, Academy of Sciences of the USSR, Moscow, 1953).

4. B. Grünbaum, Arrangements and Spreads. American Mathematical Society, Providence, RI, 1972.

5. N. J. Martinov, On conjecture 2.4 of Grünbaum. Mathematics and Education in Mathematics (Proc. 19th Spring Conference of the Union of Bulgarian Mathematicians, Sunny Beach, April 1990). Bulgarian Academy of Science, Sofia, 1990, pp. 112-117.

6. G. B. Purdy, On the number of regions determined by $n$ lines in the projective plane. Geom. Dedicata $9(1980), 107-109$.

7. P. Salamon and P. Erdös, The solution to a problem of Grünbaum. Canad. Math. Bull. 31 (1988), 129-138.

8. J. E. Taylor, Zonohedra and generalised zonohedra. Amer. Math. Monthly 99 (1992), 108-111.

Received December 10, 1990, and in revised form April 26, 1992. 